\title{
A Comprehensive Compensation Technique for Process Variations and Environmental Fluctuations in Digital Integrated Circuits
}

\author{
D. Andrade ${ }^{1}$, A. Calomarde, A. Rubio \\ Universitat Politècnica de Catalunya \\ Barcelona, Spain \\ ${ }^{1}$ dam1029@eel.upc.es
}

\begin{abstract}
Process variability and environmental fluctuations deeply affect the digital circuits performance in many different ways, one of them, the data processing time which may cause error on synchronous digital circuits due to underestimated time violations. This situation is commonly avoided adding time margins to the clock signal making it larger than nominal worstcase data process time, penalizing the global performance. In this paper a new mechanism for compensating both environmental fluctuations and process parameters variations effects on digital circuits is presented. The environmental compensation mechanism regenerates the clock signal for a pipelined system stages adding a compensated skew component depending on the local environmental conditions of every one of these stages. The process variations are corrected with a calibration circuit which adjusts the clock period in every stage taking into account its particular static deviations.
\end{abstract}

\section{INTRODUCTION}

In every semiconductors technology upgrade a several improvements are achieved, being most of them a consequence of the shrinking transistor's feature size tendency. But as long as the transistor channel length reaches tens of nanometers regime approaching to the limit of optical lithography, efficient control over the multiple devices parameters is becoming more difficult, yielding to an increase on devices mismatch, carriers mobility impact, non homogeneous doping profile, threshold voltage variability, among others. These process variabilities deviates the resulting integrated circuit from its specifications degrading its performance [1]. For example, according to the last ITRS report [2], the threshold voltage will reach a variability of $112 \%$ for 2022 which is critical, because MOS transistor electrical characteristics and behavior strongly depends on this parameter.

In addition, the power supply delivery network increases its complexity in order to provide energy to every device in highly complex chips, but the network's parasitics do not scale at the same ratio as supply voltage does and in conjunction with high fast transient currents, problems like $L \frac{d i}{d t}$, IR-Drop, ground

This research work has been supported by the Spanish Ministry of Science and Innovation (MICINN) through the project TEC2008-01856 with the additional participation of FEDER founds and the EU project TRAMS(248789). The group of research is considered a consolidated group by the MICINN. bounce, etc. increase the proportional impact on voltage level. For the incoming technologies these problems are expected to be aggravated [3] because higher parallelism of larger systems with more devices switching at same time, increase the transient current peaks magnitude and frequency and so the voltage fluctuations, making usual solutions like decoupling capacitor insufficient [4].

Similar situation is present on IC temperature fluctuations: as long as more complex systems perform more operations per second, more heat is generated inside the chip in a nonhomogeneous way. According to ITRS last report the ICs power dissipation [2] will exhibit a rather steady tendency in forthcoming years saving the chip from destructive risks, but not from the temperature gradient effects on devices characteristics, for example, on threshold voltage, adding dynamic fluctuations superimposed on its static process variations. For these reasons, two different cells on the same system could have slight temperature difference with different fluctuations profile due to its own activity and the heat transmitted from other circuits on the chip, making them have different response at the same stimulus.

Process variations are commonly addressed adding extra steps to the manufacturing process to gain a finer control over it and/or to perform corrections over some devices or entire sections of the chips. Due to its static nature, corrections aimed to process variability are performed once, but for environmental factors, the implemented solution has to follow its dynamic nature making more difficult to sense and compensate them. These efforts are getting higher design time consuming, less effective and impose higher penalization on circuits' performance.The solution proposed on this article compensate all these factors, both dynamic and static, with a comprehensive strategy based on simple built-in circuits adjustable to process variations and performing a real time sensing/compensation of dynamic environmental fluctuations.

The paper is organized as follows: in section II-A the proposed mechanism for compensate environmental factors, voltage and temperature, is presented, in section II-B how this compensation method is extended taking into account process variation with calibration extra-circuitry is introduced. The advantages of compensating mechanisms are analyzed through 
a practical example, a carry ripple adder, in section III. Finally section IV presents the conclusions of this work.

\section{COMPENSATION MECHANISMS}

The proposed compensation method is divided in two mechanisms each of them take into account the nature of the compensated phenomena: the dynamic fluctuations for temperature and voltage and the static process variability effects on circuits. The first one focused in environmental fluctuations is described in subsection II-A and the part related to process variability in subsection II-B .

\section{A. First mechanism: Local temperature and voltage fluctua- tions compensation}

The proposed temperature and voltage compensating mechanism consists in the allocation of a chain made of an even number of inverters in the clock path between the input and output registers of a given circuit as shown in Fig. 1. Both of them, the logic circuit and the inverter chain, will be merged in the same layout in order to be affected by the same temperature and voltage fluctuations defined as shown in Eq. 1 and 2 respectively

$$
\begin{aligned}
V_{n}^{\prime}(t) & =\left[V_{D D}-\Delta V_{D D}(t)\right]-\left[V_{S S}-\Delta V_{S S}(t)\right] \\
T_{m}^{\prime}(t) & =T_{m}-\Delta T_{m}(t)
\end{aligned}
$$

being $V_{n}^{\prime}(t)$ the actual voltage of the circuit, $\Delta V_{D D}(t)$ and $\Delta V_{S S}(t)$ the time dependent fluctuations on each power rail, $V_{D D}$ and $V_{S S}$ its nominal values, $T_{m}^{\prime}(t)$ the local temperature, $T_{m}$ the nominal temperature and $\Delta T_{n}(t)$ its time dependent fluctuations.

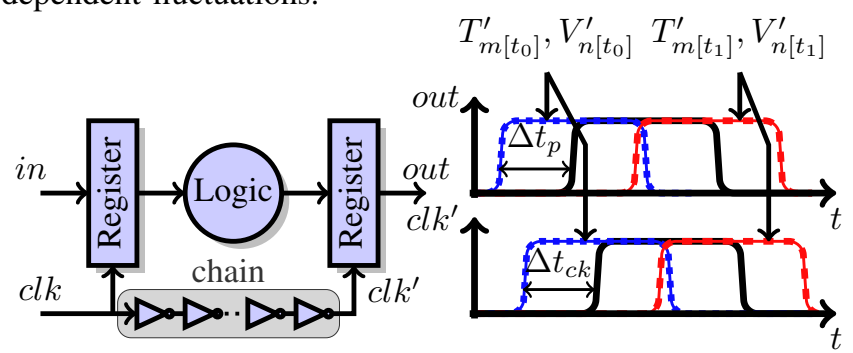

Fig. 1. Compensation for environmental factors basic principle. $\Delta t_{p}$ includes the registers timing parameters(setup and throughput time).

The length of the inverter chain is calculated to match the clock period under nominal environmental conditions (chosen according with the largest nominal data process time). Because inverter chain and logic circuit are attached to the same pair of power supply node and embedded in the same layout, every change on the environmental conditions affect them in similar way, deviating the data process time from the its nominal value in $\Delta t_{p}\left(V_{n}^{\prime}, T_{m}^{\prime}\right)$ and regenerating the clock signal with an additional skew component $\Delta t_{c k}\left(V_{n}^{\prime}, T_{m}^{\prime}\right)$, depending both of them in the characteristics of these changes. The inserted skew will be very similar to the deviation on data process time (Eq. 3), holding the time margin between the clock edge and the data arrival, as shown in Fig. 1. The chain components were chosen inverters because it can be merged in the main circuit's layout without greatly affecting it and provides fine control over the total inserted skew.

$$
\Delta t_{c k}\left(V_{n}^{\prime}, T_{m}^{\prime}\right) \simeq \Delta t_{p}\left(V_{n}^{\prime}, T_{m}^{\prime}\right)
$$

An even better performance is achieved when this mechanism is implemented in multiple stage pipelined systems as shown in Fig. 2. Considering the fluctuations on voltage and temperature in every stage non correlated (i.e., with different fluctuation profile), for the environmental conditions present in the first stage $S T_{0}$ at given time $t_{0}, V_{n 0\left[t_{0}\right]}^{\prime}, T_{m 0\left[t_{0}\right]}^{\prime}$, the clock signal $c l k_{1}$ will be regenerated with a skew dependent in these environmental conditions and ideally equal to stage data process time deviation $\Delta t_{p S T 0}$. For the next stage $S T_{1}$ with different local conditions at the same time $\left(V_{n 1\left[t_{0}\right]}^{\prime}, T_{m 1\left[t_{0}\right]}^{\prime}\right)$, the clock signal $c l k_{2}$ at the output of the second inverter chain is the algebraic sum of its own generated skew and the generated in the previous stage. In the general form, the clock signal at the $i-t h$ stage is the addition of original clock signal $c l k$ and all the generated skews $\Delta t_{p i}$ from the first stage to the current $i-t h$ stage as stated in Eq. 4.

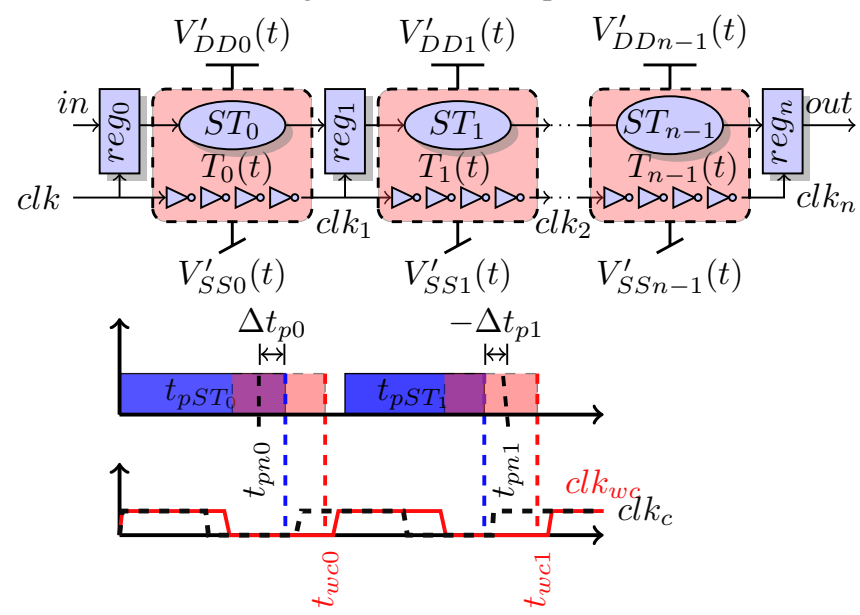

Fig. 2. Compensation for environmental factors fluctuations in multiple stage pipelined circuits. Environmental fluctuations affects both logic and inverters chain in the same way.

$$
c l k_{i}>c l k+\sum_{i=0}^{n} \Delta t_{p_{i}}\left(V_{n i}^{\prime}, T_{m i}^{\prime}\right)
$$

Because the clock regeneration follows a domino mechanism, it causes a compensation effect, due to the partial random nature of the fluctuations. In the conventional worst case design approach the clock period $c l k_{w c}$ is chosen to be larger than the largest nominal data process time $t_{p n}$ taking into account the maximum possible deviations on it $\max \Delta t_{p n}$ provoked by all the factors involved as shown in Eq. 5. But in this proposal, the clock for the $i-t h$ stage $\left(c l k_{i}\right)$ will be dynamically adapted to the instantaneous current conditions of the stage and the previous ones(Eq. 4).

$$
c l k_{w c}>t_{p n}+\max \Delta t_{p n}
$$

The compensation is performed with no additional control signals or feedback paths which would made it slower, there are not latency, do not add many extra-steps on design process 
and no extraordinary effort must be dedicated to design the compensator. For larger systems with larger number of stages better result are expected, because the time savings in every one of them allows to reduce the total clock periods needed by the system to complete data processing. The drawbacks of the technique are the overhead and extra-power consumption introduced. The resulting circuit operates locally asynchronous while the whole system operates synchronously.

In [5] a more extensive description of this mechanism is presented and more analysis and advantages are described.

\section{B. Second mechanism: Adjusting compensation for process} variability

In the first compensation mechanism just environmental factors fluctuations were considered, but static process variability affecting the inverter chain and logic circuit may introduce deviation on nominal timing parameters for both circuits, provoking the time margin between regenerated clock edge and data arrival may be reduced or enlarged. Even worse scenario may arise when environmental conditions fluctuate interacting with static variation, for example, in the case of threshold voltage which dynamically fluctuate due to its timevarying temperature dependence, as shown in Eq. 6 where $V_{t h}$ is the nominal threshold voltage and $\Delta V_{t h}\left(x y, T_{m}^{\prime}\right)$ the dynamic fluctuations from this nominal value.

$$
V_{t h}\left(x y, T_{m}^{\prime}\right)=V_{t h}+\Delta V_{t h}\left(x y, T_{m}^{\prime}\right)
$$

If process variations enlarge the time margin between data and clock, some of the inverters in the chain are unnecessarily generating extra power consumption and penalizing circuit speed; in the other hand, if time margin is too close or even exceeded by data arrival metastability or data lose may occurs. In order to avoid this situation, an adjusting mechanism is proposed which fits the number of inverters according with the effects of process variability over the circuit and inverter chain itself. The technique incorporates the scan path mechanism in order to have more controllability and observability over the input/output at every stage and enabling test and normal operation mode options. The scan path allows to load tests vectors corresponding to worst case path on each stage. When the circuit enters in the calibrating phase performs the operations shown in Fig. 3 (in the Fig. 4 the calibration block diagram is shown).

Once the final stage's calibration is accomplished, the system enters on normal operation mode with a fitted compensation circuit ready to effectively mimicking the effects of local temperature and voltage, and including the process variation too. The calibrating circuit is shown in Fig. 4 for just one stage. This process is performed once at the system start up but can be programmed with some periodicity according with the system characteristics.

Observe that after adjusting the number of inverter cells, the regenerated clock signal and data signal edges may be very close from each other and metastability may arise; to avoid it, the algorithm adds a pair of inverters to the chain even before error detection step is performed forcing the system to include

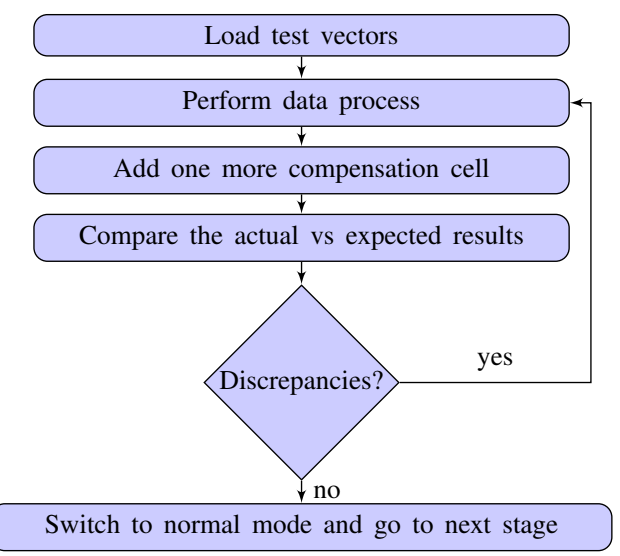

Fig. 3. Calibration algorithm to adjust the compensation according with process variations impact on devices' physical parameters

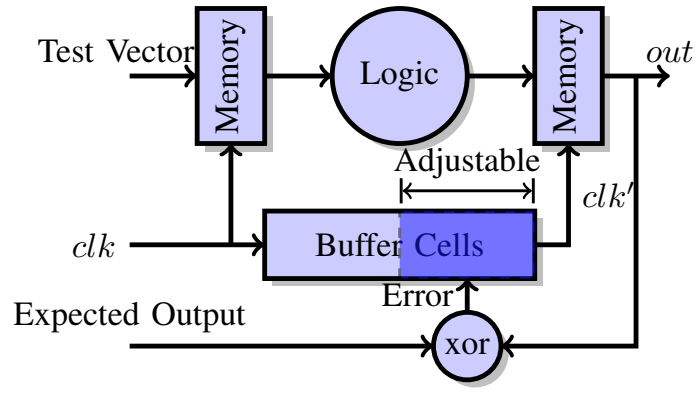

Fig. 4. The calibration of process variations is performed as shown with some extra-circuitry and profiting idle time

one additional buffer to increase the time margin between both signals. For systems where this solution are considered not sufficient, the free-error signal can be delayed a number of clock cycles as the extra buffer cells wants to be added, enlarging the time margin between data and clock signals.

\section{A PRACTICAL EXAMPLE: CARRY RIPPLE ADDER}

To illustrate the advantages of these compensation mechanisms, a carry ripple adder was chosen as testbench because its pipelined structure make easy to identify the contributions of every stage on data process time deviations. The inverter chain was embedded in the same adder basic cell(ABC) as shown in Fig. 5. Doing it in that way presents two advantages: a) this new cell can be added to technology libraries and used in automated synthesis and b) process variations and environmental fluctuations can be followed very close due to this proximity. The $\mathrm{ABC}$ nominal largest data process time corresponds to carry output $\left(C_{o}\right.$ in Fig. 5)when the input switch between $101 \Longleftrightarrow 100$. This output has been measured for a $-10 \% V_{n} \leq \Delta V_{n}^{\prime} \leq 10 \% V_{n}, 25^{\circ} C \leq T_{m}^{\prime} \leq 125^{\circ} C$ and inverters were added until Eq. 7 is fulfilled

$$
\Delta t_{p-e} \leq m \Delta t_{n o t-e}
$$

where $\Delta t_{p-e}$ stands for deviations on data process time and $\Delta t_{n o t-e}$ stands for one single not cell time fluctuations, both of them provoked by environmental fluctuations, and $m$ for the amount of inverters needed to fulfill the equation, in 
this case, using a $45 \mathrm{~nm}$ technology, $m=20$. The resulting time fluctuations profiles are presented in Fig. 6, where all values have been normalized using the $\mathrm{ABC} t_{p}$ under nominal conditions $\left(T_{m}\right.$ and $\left.V_{n}\right)$. It must regarded for the whole temperature and voltage range of fluctuations, the inverter chain effectively follows the ABC time deviations blocking time violations cases. The resulting compensated $\mathrm{ABC}$ version

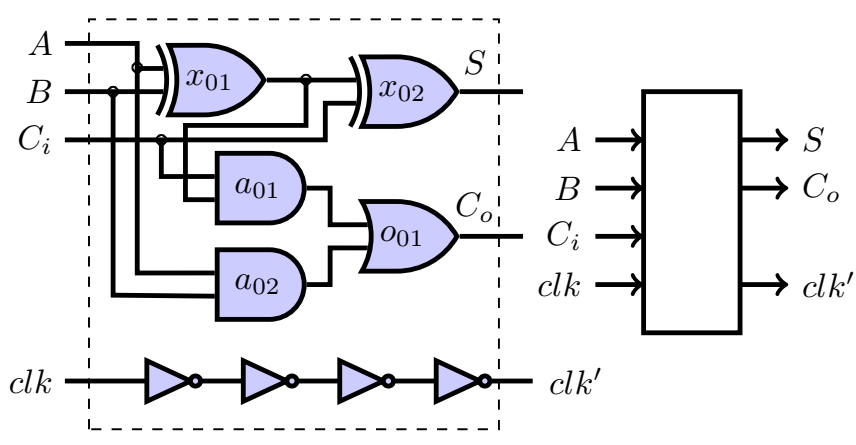

Fig. 5. Adder basic cell (ABC) used in simulations
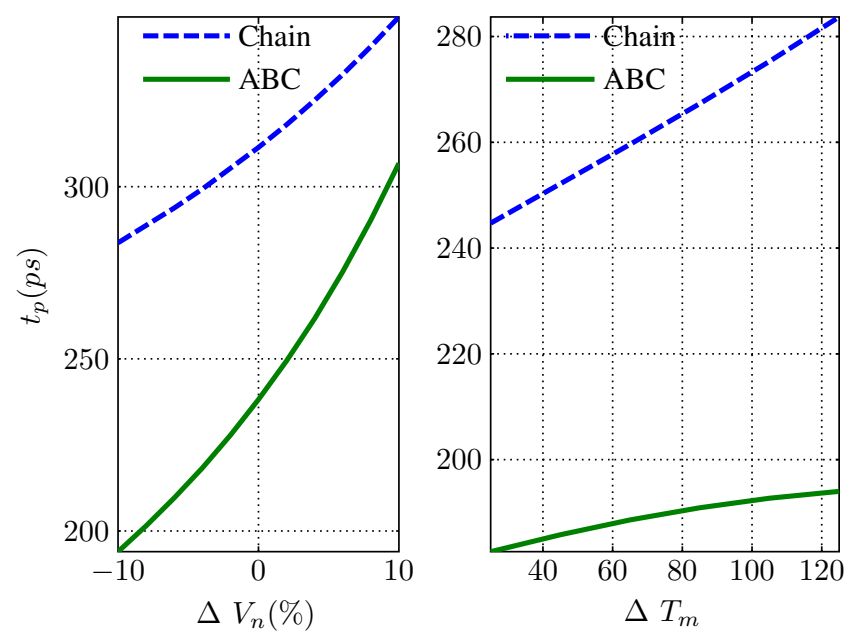

Fig. 6. Environmental fluctuation effects on the compensated cell. The right graph is for voltage fluctuations when $T_{m}^{\prime}=125^{\circ} \mathrm{C}$ and left graph is for Temperature fluctuations when $V_{n}^{\prime}=V_{n}-10 \% V_{n}$.

enlarge the layout area by a factor of almost 2.1 for a demifull-custom approach. With this compensated ABC a 16-bit Carry Ripple Adder was implemented. The inverter chain must be adjusted in order to fit the deviations provoked by process variation and avoid its effects on the time margin effectively hold by the inverter chain. In this particular case, the adjust mechanism need to check just the most significant bit correctness and add inverter pairs until the condition stated in Eq. 8 is fulfilled

$$
n \cdot t_{p}+\sum_{i=1}^{n} \Delta t_{p-p} \leq\left[n \cdot t_{c}+\sum_{i=1}^{n} \Delta t_{c-p}\right]+a \cdot t_{n o t}
$$

where $n$ is the number of bits, $t_{p}$ is the nominal ABC process time, $\Delta t_{p-p}$ are the deviations due to process variations, $t_{c}$ is the time for the inverter chain embedded into the basic cell,
$\Delta t_{c-p}$ are the deviations on this time due to process variations, $\Delta t_{n o t}$ is the not process time and finally, $a$ is the number of needed cell to equal both side of the equation that may be positive or negative depending on the effects of process variations over the circuits. For this example, Monte-Carlo simulation has been performed and the process variations can be effectively compensated modifying the amount of inverters per $\mathrm{ABC}$ within the range of $18 \leq 20 \leq 24$.

\section{CONCLUSIONS}

In this paper we have introduced a strategy to compensate environmental and process variations, key factors limiting the performance of ICs built from modern CMOS technologies. The strategy is based on the implementation of a clock regenerating mechanism: a chain of inverters reproduces the clock signal for the receiving latches of a circuit stage. As inverters are affected by the same environmental fluctuations as the stage's processing logic, an efficient compensation is performed, allowing reductions on clock safety margins improving the circuit performance. The mechanism is calibrated to compensate process variations effects in the circuit.

\section{REFERENCES}

[1] M. Orshansky, L. Milor, P. Chen, K. Keutzer, and C. Hu, "Impact of spatial intrachip gate length variability on the performance of highspeed digital circuits," Computer-Aided Design of Integrated Circuits and Systems, IEEE Transactions on, vol. 21, pp. 544-554, May 2002.

[2] ITRS, "Executive summary," tech. rep., ITRS, 2007.

[3] K. Shakeri and J. D. Meindl, "Compact physical ir-drop models for chip/package co-design of gigascale integration (gsi)," IEEE Transaction on Electon Devices, vol. 52, pp. 1087-1096, June 2005.

[4] H. Yamamoto and J. Davis, "Decreased effectiveness of on-chip decoupling capacitance in high-frequency operation," Very Large Scale Integration (VLSI) Systems, IEEE Transactions on, vol. 15, pp. 649-659, June 2007.

[5] D. Andrade, F. Martorell, A. Calomarde, F. Moll, and A. Rubio, "A new compensation mechanism for environmental parameter fluctuations in cmos digital ic's," Microelectronics Journal, vol. 40, pp. 952-957, June 2009. 